\title{
DYNAMIC ROUTING IN QOS-AWARE TRAFFIC ENGINEERED NETWORKS
}

\author{
Stefano Avallone, ${ }^{1}$ Fernando Kuipers, ${ }^{2}$ Giorgio Ventre, ${ }^{1}$ and Piet Van Mieghem ${ }^{2}$ \\ ${ }^{1}$ COMICS Lab \\ Dipartimento di Informatica e Sistemistica \\ Universita di Napoli Federico II \\ Via Clandio 21, 80125 Napoli, Italy \\ \{stavallo, giorgio\}@unina.it \\ ${ }^{2}$ Network Architectures and Services \\ Faculty of Electrical Engineering, Mathematics and Computer Science \\ Delft University of Technology \\ P.O. Box 5031, 2600 GA Delft, The Netherlands \\ \{F.A.Kuipers, P.VanMieghem\}@ewi.tudelft.nl
}

\begin{abstract}
We propose a proper length function for an existing QoS routing algorithm (SAMCRA) that attempts to optimize network utilization while still offering QOS guarantees. This paper presents a comparison between several proposed algorithms via simulation studies. The simulations show that SAMCRA with a proper length performs similarly or even better than the best among the other algorithms and it has a fast running time.
\end{abstract}

Keywords: QoS routing, Traffic Engineering, network dynamics

\section{Introduction}

The Internet research community is making a great effort in order to define efficient network management and control functions. The driving forces behind this effort are new applications with specific performance requirements. For instance, real-time applications need delay and jitter guarantees, while a financial transaction must have low or virtually no packet loss. By offering service differentiation combined with the maximization of throughput, ISPs can increase their revenues. The challenge is therefore to define a routing algorithm which meets the users' requirements and which optimizes network resources.

Many algorithms [Kuipers et al., 2002] have been proposed to find the shortest path subject to multiple constraints. This problem, called MCOP (Multi- 
Constrained Optimal Path), is NP-complete. Therefore, although there exist exact algorithms such as SAMCRA [Van Mieghem and Kuipers, 2004], mainly heuristics have been proposed for this problem. Such algorithms usually do not address the maximization of the throughput and the number of admitted calls. Instead, optimizing these parameters is the goal of another class of algorithms, denoted as traffic engineering algorithms. While focused on the behaviour in a dynamic scenario, most of them do not take into account additive QoS constraints and only consider bandwidth.

In this paper we present a routing scheme that aims at maximizing throughput (or minimizing blocking), while satisfying the users' QoS requirements. It is our goal to combine these two objectives as efficiently as possible. We propose to use SAMCRA [Van Mieghem and Kuipers, 2004] with a special path length definition that guarantees the QoS constraints and accounts for the traffic engineering objectives. For clarity, we name this variant SAMCRA-B.

The performance of SAMCRA-B and several other algorithms is evaluated through simulations. All the considered algorithms do not make use of any a-priori knowledge about either predicted traffic or future demands. In the literature, such algorithms are denoted as online. We assume the knowledge of quasi-static information such as the network topology and the set of ingressegress nodes of the network. The only dynamic information is the residual bandwidth (i.e. the portion of the link capacity not yet reserved) of each link in the network.

This paper is structured as follows. In Section 2 we give a formal definition of the considered routing problem. Some solutions for routing bandwidthguaranteed paths are discussed in Section 3. Section 4 overviews SAMCRA and the choice of a proper length function. The performance studies are shown in Section 5. Section 6 concludes our work.

\section{Problem Statement}

The network is modelled as a graph $G(N, E)$, where $N$ is the set of nodes and $E$ is the set of links. With a slight abuse of notation we will also denote by $N$ and $E$, respectively, the number of nodes and the number of links. Each link $l \in E$ is assigned an $(m+1)$-dimensional QoS link weight vector $\vec{w}(l)=$ $\left[w_{0}(l), w_{1}(l), \ldots w_{m}(l)\right]$, where $w_{0}(l)$ is the available bandwidth on link $l$ and the other components are the values of $m$ additive QoS measures. Additionally, the capacity of a link $l$ is denoted by $C(l)$.

A flow request is defined by a triple $(s, d, \vec{Q})$, where $s$ is the source node, $d$ is the destination node and $\vec{Q}=\left[Q_{0}, Q_{1}, \ldots Q_{m}\right]$ is a vector representing its QoS requirements. Specifically, $Q_{0}$ is the requested bandwidth while the other components are constraints on the values of the additive QoS measures along the path. Even though minimum (maximum) QoS constraints can be 
easily treated by omitting all links which do not satisfy the requirement, we explicitly consider available bandwidth due to its central role played in resource optimization strategies. Multiplicative QoS measures are disregarded because, if we assume independent measures over the links, we can transform them into additive QoS measures by taking the logarithm [Van Mieghem and Kuipers, 2004].

When a flow request arrives, the routing algorithm searches for a feasible path $P$ that obeys:

$$
\left\{\begin{array}{l}
w_{0}(P) \stackrel{\text { def }}{=} \min _{l \in P} w_{0}(l) \geq Q_{0} \\
w_{i}(P) \stackrel{\text { def }}{=} \sum_{l \in P} w_{i}(l) \leq Q_{i}, \quad \forall i=1, \ldots m
\end{array}\right.
$$

In case no feasible path is found, the request is rejected. In presence of multiple feasible paths, the algorithm chooses the one which is thought to optimize network utilization. Typically, a path length function is defined and the feasible path with the smallest length is selected.

\subsection{Discussion on QoS link weights}

This subsection discusses the setting of QoS link weights $(\vec{w}(l))$ in a dynamic scenario. The guideline is the fulfillment of the QoS requirements of the flows. It is safe to state that the link weight associated with the available bandwidth should be as close as possible to the current bandwidth availability. As far as additive link weights, a path $P$ returned by an exact algorithm is such that $\sum_{l \in P} w_{i}(l) \leq Q_{i}, i=1, \ldots m$. But, the QoS requirements of a flow are satisfied if the perceived QoS is within the constraints, i.e. $\sum_{l \in P} q_{i}(l) \leq Q_{i}, i=1, \ldots m$, where $q_{i}(l)$ is the value of the $i$-th QoS measure experienced crossing link $l$.

Assume to set the additive QoS link weights $\left(w_{i}(l)\right)$ equal to the current experienced values (i.e. $q_{i}(l)$ ). Hence $\sum_{l \in P} q_{i}(l)=\sum_{l \in P} w_{i}(l) \leq Q_{i}$ and the QoS constraints are met. But, as a consequence of routing new flows on links of $P$, the actual QoS values $q_{i}(l)$ deteriorate and therefore the QoS granted to already admitted flows may not be preserved. Instead, we assume that the QoS link weights are constant and independent of the current link status $\left(q_{i}(l)\right)$. Their value is an upper bound to the actual QoS value, in the sense that if the allocated bandwidth is less than the link capacity, then the QoS values experienced by packets crossing the link do not exceed the QoS weights. This assures that $\sum_{l \in P} q_{i}(l) \leq \sum_{l \in P} w_{i}(l) \leq Q_{i}, i=1, \ldots, m$, i.e. the additive QoS constraints will be satisfied even after new flows are routed.

\section{Existing Traffic Engineering Algorithms}

Among the earliest proposed algorithms, widest-shortest path [Guerin et al., 1997] (labeled as IWSP(MinHop)"throughout this paper) selects the path with 
the minimum hop count among all paths having sufficient residual bandwidth. If there are several such paths, the one with the maximum residual bandwidth is selected.

Most recently proposed algorithms are inspired by the work of Kar, Kodialam and Lakshman [Kar et al., 2000]. They presented an online routing algorithm (MIRA) based on the concept of minimum interference. The amount of interference on a particular source-destination pair $(s, d)$ due to routing a flow between some other source-destination pair is defined as the decrease in the maxflow between $s$ and $d$. The maxflow [Ahuja et al., 1993] value is an upper bound on the total amount of bandwidth that can be routed between two edge nodes. The minimum interference path between a particular source-destination pair is the path which maximizes the minimum maxflow between all other source-destination pairs. The idea is that a new request must follow a path that does not interfere excessively" with a route that may be critical to satisfy a future demand. The problem of finding the minimum interference path is proved to be NP-hard. Therefore, Kar et al. [Kar et al., 2000] proposed to determine appropriate link costs, prune links with insufficient available bandwidth and compute the shortest path in the pruned topology.

Wang et al. [Wang et al., 2002] proposed a different definition for link costs in MIRA. We denote this variant of MIRA as "NewMIRA". The performance evaluation in [Wang et al., 2002] shows that, in a dynamic scenario, NewMIRA outperforms MIRA.

Banerjee and Sidhu [Banerjee and Sidhu, 2002] proposed two algorithms: TE-B, which takes into account only a bandwidth requirement, and TE-DB, which considers also a delay constraint. The authors introduced three objectives for traffic engineering: (a) reducing the blocking of flows, (b) minimizing network cost and (c) distributing network load. This formulation has three objective functions (plus the delay constraint in the case of TE-DB) and is proved to be NP-complete [Banerjee and Sidhu, 2002]. Banerjee and Sidhu presented another formulation in which objective functions (a) and (b) are transformed into constraints. Both TE-B and TE-DB use TAMCRA [De Neve and Van Mieghem, 2000], the predecessor of SAMCRA [Van Mieghem and Kuipers, 2004], to find a set of $k$ paths satisfying the set of constraints and then select the one with the shortest length according to (c).

Iliadis and Bauer [Iliadis and Bauer, 2002] introduced a new class of routing algorithms, called SMIRA (simple minimum-interference routing algorithms). These algorithms evaluate the interference on a source-destination pair by means of a $k$-shortest-path-like computation instead of a maxflow computation. The set of $k$ paths between a source-destina-tion pair $(s, d)$ is determined by first computing the widest-shortest path [Guerin et al., 1997] between $s$ and $d$. Then, all the links along this path with a residual bandwidth equal to the bottleneck bandwidth of the path are pruned. The second path is the widest- 
shortest path in the pruned topology. This procedure is repeated until either $k$ paths are found or no more paths are available. The cost of links belonging to the set of $k$ paths is increased proportionally to the weight of the path and the ratio of bottleneck bandwidth to residual bandwidth. Iliadis and Bauer [lliadis and Bauer, 2002] proposed two algorithms, MI-BLA and MI-PA. The simulations in [Iliadis and Bauer, 2002] show that MI-PA outperforms MI-BLA.

\section{SAMCRA}

To guarantee QoS constraints and optimize network resource usage, we decided to use SAMCRA [Van Mieghem and Kuipers, 2004] with a new path length function and to study its behaviour in a dynamic scenario. First, we briefly review the basic concepts on which SAMCRA relies. The length of a path $P$ proposed in [Van Mieghem and Kuipers, 2004] is a non-linear function of the $m$ additive QoS measures it considers:

$$
L(P)=\max _{1 \leq i \leq m} \frac{w_{i}(P)}{Q_{i}}
$$

so that path $P$ satisfies the constraints when $L(P) \leq 1$. An important corollary of a non-linear path length is that the subsections of shortest paths in multiple dimensions are not necessarily shortest paths. This necessitates a $k$-shortest path approach, which is essentially Dijkstra's algorithm that does not stop when the destination is reached, but continues until the destination has been reached $k$ times. Not all sub-paths are stored, but an efficient distinction based on non-dominance is made: a (sub)-path $P_{1}$ is dominated by a (sub)-path $P_{2}$ if $w_{i}\left(P_{2}\right) \leq w_{i}\left(P_{1}\right)$ for $i=1, \ldots, m$, with an inequality for at least one link weight component $i$. SAMCRA only considers non-dominated (sub)-paths.

We refer for more details on the above concepts, possible improvements and an implementation of the algorithm to [Van Mieghem and Kuipers, 2004]. Here, we explain the above concepts through a simple example. Like Dijkstra's algorithm, SAMCRA starts from the source node and explores the neighboring nodes while moving toward the destination node. Unlike Dijkstra, SAMCRA may have to store more than only the shortest sub-path for each visited node. To explain this point, consider the simple network of Figure 1. The vector $\vec{w}(l)=\left[w_{0}(l), \ldots w_{3}(l)\right]$ of QoS link weights is shown around each link. SAMCRA does not consider the available bandwidth constraint, but it suffices to prune from the network graph all the links with insufficient available bandwidth and run the algorithm on the reduced graph. Suppose SAMCRA has to route a flow from $A$ to $G$ subject to the QoS constraint vector $Q=[5,14,11,22]$. Three sub-paths are available from the source node $A$ to the intermediate node $E$. The lengths of those sub-paths are:

$$
L\left(P_{A B E}\right)=\max \frac{4+3}{14}, \frac{1+7}{11}, \frac{7+1}{22}=0.73
$$




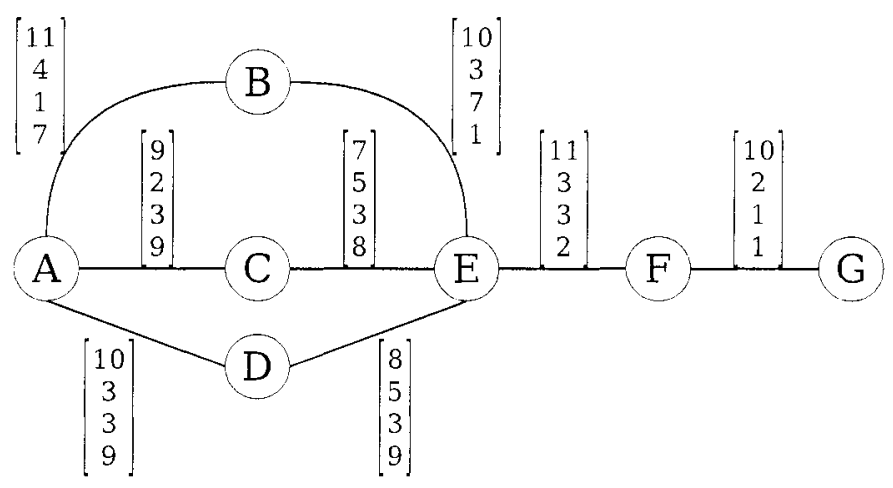

Figure 1. Simple network to illustrate SAMCRA's principles

$$
\begin{aligned}
& L\left(P_{A C E}\right)=\max \frac{2+5}{14}, \frac{3+3}{11}, \frac{9+8}{22}=0.77 \\
& L\left(P_{A D E}\right)=\max \frac{3+5}{14}, \frac{3+3}{11}, \frac{9+9}{22}=0.82 .
\end{aligned}
$$

The lengths of their corresponding paths from $A$ to $G$ are:

$$
\begin{aligned}
& L\left(P_{A B E F G)}=\max \frac{12}{14}, \frac{12}{11}, \frac{11}{22}=1.09\right. \\
& L\left(P_{A C E F G}\right)=\max \frac{12}{14}, \frac{10}{11}, \frac{20}{22}=0.91 \\
& L\left(P_{A D E F G}\right)=\max \frac{13}{14}, \frac{10}{11}, \frac{21}{22}=0.95 .
\end{aligned}
$$

$P_{A B E}$, is the shortest sub-path, but it leads to a non-feasible path, since $L\left(P_{A B E F G}\right)>1$. If the algorithm stores just the shortest sub-path in the intermediate nodes, it erroneously concludes that a feasible path does not exist (the two other paths are feasible). In order to reduce complexity (while still returning the exact solution), SAMCRA does not store all the sub-paths but discards the dominated ones. In the example, sub-path $P_{A D E}$ is dominated by $P_{A C E}$ since $w_{i}\left(P_{A C E}\right) \leq w_{i}\left(P_{A D E}\right)$ for $i=1,2,3$. Given (1) as path length function and non-negative QOS link weights, sub-path $P_{A D E}$ can be safely discarded.

As discussed in [Van Mieghem and Kuipers, 2004] SAMCRA can be used with any path length function, but the definition (1) is a function of the QoS link weights $\left(w_{i}(l), i=1, \ldots m\right)$ and of the QoS constraints $\left(Q_{i}, i=1, \ldots m\right)$. As discussed in Section 2.1, static QoS link weights should be considered. Thus, if link weights are load-independent, the path length function (1) does not take into account network utilization. We expect that a better dynamic behavior can 
be achieved by letting the path length be a function of dynamic information such as the available bandwidth. We assume:

$$
L(P)=\sum_{l \in P} c(l)
$$

where $c(l)$ is a link cost that depends on dynamic information related to link $l$. For clarity we name this variant SAMCRA-B. It selects the shortest path according to (2) among those satisfying the QoS constraints. Using such a path length function requires to add one more parameter to the dominance check. SAMCRA-B can discard a sub-path $P_{1}$ when there exists a sub-path $P_{2}$ such that $w_{i}\left(P_{2}\right) \leq w_{i}\left(P_{1}\right)$ for $i=1, \ldots m$ and $L\left(P_{2}\right) \leq L\left(P_{1}\right)$. To clarify why, consider again the example shown in Figure 1 and compute the lengths (according to (2)) of the three sub-paths from $A$ to $E$ and the corresponding paths from $A$ to $G$ using, for instance, $c(l)=\frac{1}{w_{0}(l)}$, i.e. the cost of a link is the reciprocal of the available bandwidth:

$$
\begin{aligned}
& L\left(P_{A B E}\right)=\frac{1}{11}+\frac{1}{10}=0.19 \\
& L\left(P_{A C E}\right)=\frac{1}{9}+\frac{1}{7}=0.25 \\
& L\left(P_{A D E}\right)=\frac{1}{10}+\frac{1}{8}=0.23
\end{aligned}
$$

The lengths of their corresponding paths from $A$ to $G$ are:

$$
\begin{aligned}
& L\left(P_{A B E F G)}=\frac{1}{11}+\frac{1}{10}+\frac{1}{11}+\frac{1}{10}=0.38\right. \\
& L\left(P_{A C E F G}\right)=\frac{1}{9}+\frac{1}{7}+\frac{1}{11}+\frac{1}{10}=0.44 \\
& L\left(P_{A D E F G}\right)=\frac{1}{10}+\frac{1}{8}+\frac{1}{11}+\frac{1}{10}=0.42
\end{aligned}
$$

$P_{A B E}$ is the shortest sub-path but leads to path $P_{A B E F G}$, which is not feasible because $w_{2}\left(P_{A B E F G}\right)>Q_{2}$. Thus, the algorithm cannot store just the shortest sub-path in each intermediate node. On the other hand, disregarding the condition on the length of the sub-paths would cause sub-path $P_{A D E}$, which leads to the shortest feasible path $P_{A D E F G}$, to be discarded in favour of $P_{A C E}$. Instead, by also comparing path lengths it is still possible to achieve a correct search space reduction and return the shortest path according to (2) within the constraints. Indeed, sub-path $P_{A D E}$ is not dominated by $P_{A C E}$ because $L\left(P_{A C E}\right)>L\left(P_{A D E}\right)$ and therefore it is not discarded.

The worst-case complexity of SAMCRA is $O\left(k N \log (k N)+k^{2} m E\right)$, where $k$ is the maximum amount of simultaneously stored paths. In [Van Mieghem and Kuipers, 2004], it is shown that if the QoS link weights $w_{i}(l)$ and the QoS 
constraints $Q_{i}$ are integers, SAMCRA has a pseudo-polynomial-time complexity. The complexity of SAMCRA-B is that of SAMCRA, apart from a larger value of $k$ because SAMCRA-B has to check one more condition and therefore less paths can be discarded.

\subsection{Link cost function}

This section illustrates the link cost function we introduce to improve the dynamic behavior of SAMCRA-B with respect to SAMCRA. The link cost $c(l)$ is defined as a function $\gamma$ of the link utilization $\rho=\frac{C(l)-w_{0}(l)}{C(l)}$ (ratio of the reserved bandwidth to the total capacity):

$$
\gamma(\rho)= \begin{cases}\frac{\gamma(\Delta)-1}{\Delta} \rho+1 & \text { if } \rho \leqslant \Delta \\ \gamma(\Delta) \frac{1-\Delta}{1-\rho} & \text { otherwise }\end{cases}
$$

The function $\gamma(\rho)$, depicted in Fig. 2, depends on two design parameters, $\Delta$

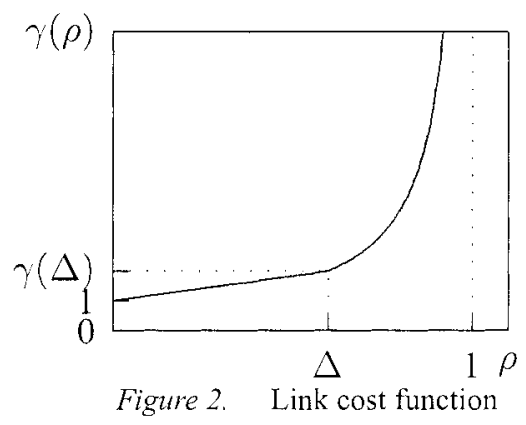

and $\gamma(\Delta)$. The rationale behind such proposal is that a minimum-hop path approach is preferable when network load is low, since it prevents longer paths from consuming more resources. On the other hand, when network load is high, it is preferable to use the path with the maximum available bandwidth. Consuming all the available bandwidth on some links could cause future requests to be blocked. Such a twofold behavior may be achieved by appropriately setting each link cost depending on its resource occupation. The link cost function we propose is divided in two segments whose junction takes place in $(\Delta, \gamma(\Delta))$. For values of the link utilization $\rho$ less than $\Delta$, the link cost grows linearly. As soon as $\rho$ becomes greater than $\Delta$, the link cost tends to infinity as $\rho$ approaches 1 . With such a function, link costs are only slightly differing for low traffic loads. Low slope values of the linear segment let the routing algorithm behave similarly to the minimum-hop path routing. Instead, small load variations reflect in substantial link cost differences as soon as the load on the link increases beyond a specified threshold $\Delta$. In this way, links having more available bandwidth are highly preferable. 
Table 1. Random variables used to specify network flows

\begin{tabular}{lc}
\hline Name & Description \\
\hline IntArriv & Inter-arrival time between two successive flow requests \\
Source & Source node \\
Dest & Destination node \\
FlowDur & Flow duration \\
Bwd & Requested bandwidth \\
QoS & $i$-th additive QoS constraint \\
\hline
\end{tabular}

The values assigned to $\Delta$ and $\gamma(\Delta)$ determine the relative importance of the two approaches. We expect that their "optimal" values are dependent on the network topology and the traffic load. As an attempt, we have used $\Delta=0.6$ and $\gamma(\Delta)=1.5$ for the simulations shown in the next section. From now on, we will implicitly consider (3) as the link cost function of SAMCRA-B.

\section{Performance Studies}

The performance studies of this section aim to compare the algorithms based on the minimum interference concept (New MIRA, TE-DB, MI-PA) to SAMCRA and SAMCRA-B and to evaluate the possible gain achieved by using the new path length function (3). The experiments were carried out on several topologies generated by BRITE [Medina et al., 2000]. We used two routerlevel models, Barabasi-Albert and Waxman. All the topologies have 100 nodes and a different number of links per new node. For each topology, 10 nodes are randomly chosen to act as edge routers, the entry and exit points for the network traffic. The capacity of the links is uniformly distributed between 100 and 1024 units. We considered two additive QoS constraints $(m=2)$, the first uniformly distributed between 3 and 8 units, while the second $\left(w_{2}(l)\right)$ uniformly distributed between 4 and 9 units. All links are symmetric, with respect to both capacity and QoS link weights.

We have developed a flow-level simulatorto analyze and compare the performance of different routing algorithms in a dynamic scenario. Our simulator makes use of several random variables to specify the characteristics of network flows (Table 1). For all the presented simulations, source and destination nodes are chosen uniformly among the set of edge nodes. We studied the performance of the routing algorithms in the generated topologies under different loads. Each test was repeated 20 times with different seeds for the random variables. For each of these 20 iterations, the algorithms under evaluation faced the same set of flow requests. Each iteration involved the generation of 120000 flows. The first 20000 were not considered in our analysis, as they 
represent a warm-up period needed by the network in order to reach a steady state regime.

For each iteration we computed the call blocking rate (CBR) and the bandwidth blocking rate (BBR) achieved by each algorithm:

$$
C B R=\frac{\text { number of rejected flows }}{\text { total number of flows }} \quad B B R=\frac{\sum_{\text {rejected flows }} \text { requested bandwidth }}{\sum_{\text {all the flows }} \text { requested bandwidth }} .
$$

We also computed the throughput after the processing of each new flow request as the sum of the bandwidth requested by the flows crossing the network at that time. In order to get a smooth throughput curve, we first computed the mean over each window of 5000 throughput samples for each iteration and then the average of the corresponding values obtained from the 20 iterations. Finally, we measured the average processor time spent by each algorithm to select a path.

\subsection{Large QoS constraints}

The purpose of this subsection is to compare all the algorithms from the viewpoint of resource optimization. Since some of them select a path disregarding additive QoS constraints, we chose the QoS constraints large such that all algorithms can return a path that obeys these constraints. We have carried out a number of simulations using several topologies and different loads. In this subsection we illustrate three different tests that are representative of the different cases we observed. Table 2 shows how we set the random variables that specify a flow and the model and the number of links of the topologies we used. The results are presented in Figures $3 \mathrm{a}-3 \mathrm{f}$. For each algorithm, we computed the mean $\mu$ and the standard deviation $\sigma$ of the CBR from 20 iterations. Each bar shown in Figures $3 \mathrm{a}, 3 \mathrm{c}$ and $3 \mathrm{e}$ represents the interval $(\mu-\sigma, \mu+\sigma)$ related to the CBR achieved by each algorithm. In tests 1 and 2 (Figures $3 a$ and $3 c)$, SAMCRA-B achieves respectively a slightly larger CBR than the minimum and the minimum CBR. The CBR of New Mira and TE-DB is higher (around 30\%) than the minimum, so as that of SAMCRA (below 10\%). In test 3 (Figure 3e), instead, the minimum CBR is achieved by New MIRA, closely followed by TE-DB and SAMCRA-B. In all the simulations we have carried out SAMCRA-B achieves the minimum CBR or a CBR close to the minimum. The bandwidth blocking rate results (not shown here) are similar to CBR ones, except that the algorithms based on the maxflow concept (New MIRA and TEDB) perform better in terms of BBR than CBR. This behaviour suggests that New MIRA and TE-DB accept those flows with larger bandwidth requirement.

While the CBR plot shows a mean value over all the iterations, the average throughput plot gives us information on the average behaviour during an iteration. Figures $3 \mathrm{~b}, 3 \mathrm{~d}$ and $3 \mathrm{f}$ indicate that the behavior of the algorithms from the 


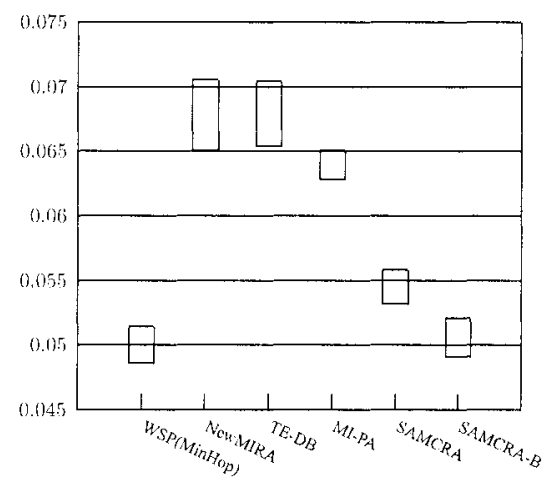

Figure $3 a$. Test 1 - Call blocking rate

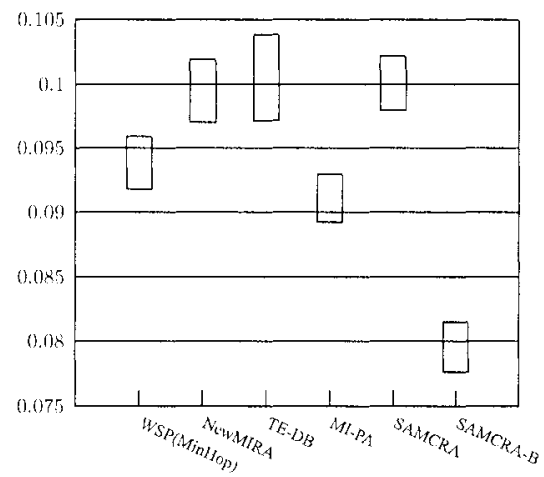

Figure 3c. Test 2 - Call blocking rate

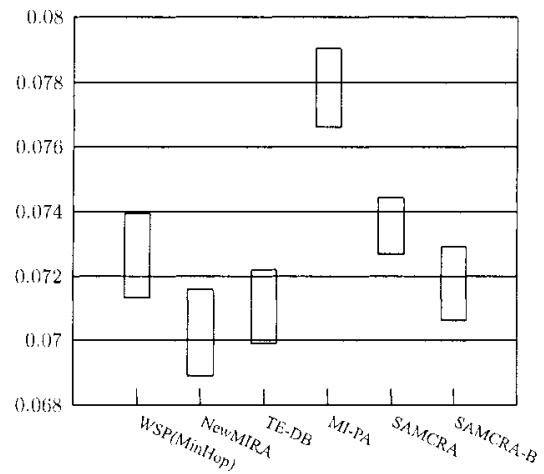

Figure 3e. Test 3 - Call blocking rate

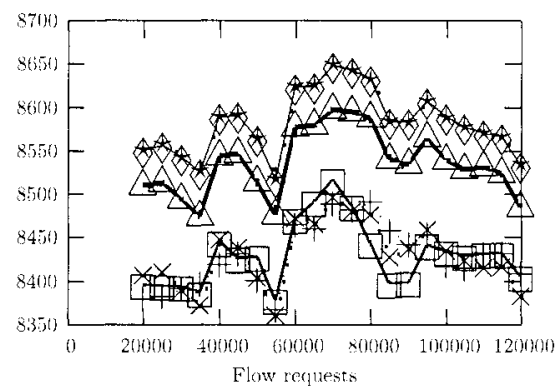

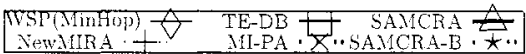

Figure 3b. Test 1 - Throughput

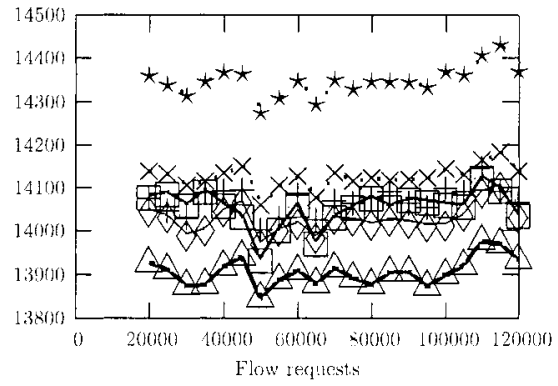

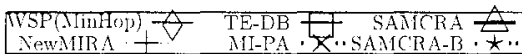

Figure 3d. Test 2 - Throughput

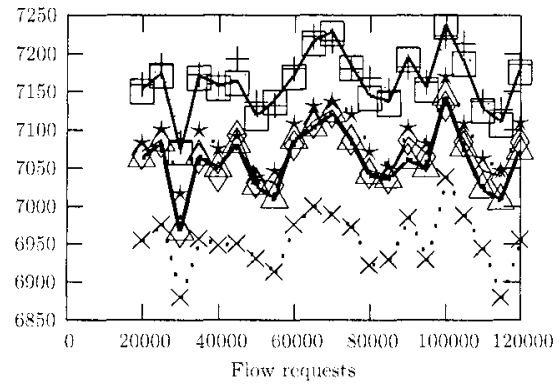

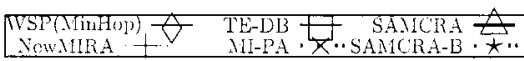

Figure 3f. Test 3 - Throughput 
Table 2. Scenario 1: flow and topology parameters

\begin{tabular}{lccc}
\hline Test & 1 & 2 & 3 \\
\hline IntArriv & $\operatorname{Exp}(1 / 0.15)$ & $\operatorname{Exp}(1 / 0.11)$ & $\operatorname{Exp}(1 / 0.5)$ \\
FlowDur & $\operatorname{Exp}(1 / 250)$ & $\operatorname{Exp}(1 / 320)$ & $\operatorname{Exp}(1 / 175)$ \\
Bwd & $\mathrm{U}(1,10)$ & $\mathrm{U}(1,10)$ & $\mathrm{U}(1,10) \quad$ with $P=0.75$ \\
QoS $_{1}$ & & $\mathrm{U}(792,800)$ & $\mathrm{U}(80,100) \quad$ with $P=0.25$ \\
QoS $_{2}$ & & $\mathrm{U}(891,900)$ & \\
\hline Topology & Barabasi & Waxman & Barabasi \\
Links & 294 & 200 & 197 \\
\hline
\end{tabular}

viewpoint of throughput is similar to that in terms of CBR. In the sense that the algorithm achieving the minimum CBR also presents the maximum throughput. Throughput plots enable to ascertain that the difference in performance between algorithms is maintained during the whole iteration. The analysis of the average computation times reveals that SAMCRA approximately requires the same time $\left(\approx 5 \cdot 10^{-4} \mathrm{~s}\right)$ as widest-shortest path. As expected, due to the less efficient search space reduction, SAMCRA-B $\left(\approx 9 \cdot 10^{-4} \mathrm{~s}\right)$ is slower than SAMCRA. Instead, the time required by New MIRA and TE-DB $\left(\approx 6 \cdot 10^{-2} \mathrm{~s}\right)$ is two orders of magnitude larger than that of SAMCRA.

\subsection{Tight QoS constraints}

Table 3. Scenario 2: flow parameters

\begin{tabular}{|c|c|c|c|c|c|}
\hline Test & $4 a$ & $4 b$ & $4 c$ & $4 d$ & $4 \mathrm{e}$ \\
\hline IntArriv & \multicolumn{5}{|c|}{$\operatorname{Exp}(1 / 0.33)$} \\
\hline FlowDur & \multicolumn{5}{|c|}{$\operatorname{Exp}(1 / 200)$} \\
\hline Bwd & \multicolumn{4}{|c|}{$\begin{array}{ll}\mathrm{U}(1,10) & \text { with } P=0.75 \\
\mathrm{U}(80,100) & \text { with } P=0.25\end{array}$} & \\
\hline$Q_{0} S_{1}$ & $U(792,800)$ & $\mathrm{U}(30,50)$ & $\mathrm{U}(24,44)$ & $\mathrm{U}(22,42)$ & $\mathrm{U}(21,41)$ \\
\hline $\operatorname{QoS}_{2}$ & $\mathrm{U}(891,900)$ & $\mathrm{U}(35,55)$ & $\mathrm{U}(29,49)$ & $U(27,47)$ & $\mathrm{U}(26,46)$ \\
\hline
\end{tabular}

This set of simulations aims at comparing the algorithms which select a path taking explicitly into consideration additive QoS constraints: SAMCRA, SAMCRA-B and TE-DB. They are evaluated from the viewpoint of resource optimization as the QoS constraints become more stringent. We present five tests, which differ only in the QoS constraints (see Table 3). The topology is the same as that used in test 2 . Figures $4 a-4 b$ shows the plots representing 


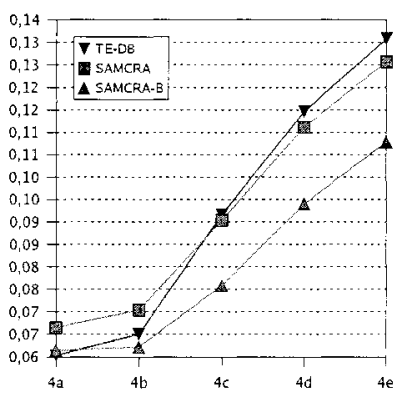

Figure 4a. Call blocking rate

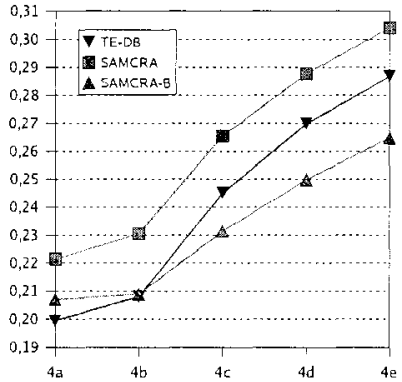

Figure $4 b$. Bandwidth blocking rate

the average call and bandwidth blocking rates for each test. When QoS constraints are large enough (test $4 a$ ), the minimum CBR is achieved by TE-DB. But, as the QoS constraints become more stringent, SAMCRA-B performs the best. Moreover, the gap between the average CBR of SAMCRA-B and those of SAMCRA and TE-DB increases. This suggests that SAMCRA-B is less sensitive to the tightening of QoS constraints than SAMCRA and TE-DB are. Also, the CBR of SAMCRA is initially larger than that of TE-DB, but eventually it becomes smaller. This suggests that SAMCRA, too, is less sensitive to the tightening of QoS constraints than TE-DB. The same conclusions can be drawn from the viewpoint of BBR (Figure $4 \mathrm{~b}$ ). Figure $4 \mathrm{~b}$ also confirms our insight regarding the fact that TE-DB performs better from the viewpoint of $B B R$ than CBR. Finally, the path computation times are similar to those indicated in the previous section.

\section{Conclusions}

We proposed a new path length function for SAMCRA and carried out simulation studies in order to compare SAMCRA-B to previous algorithms for dynamically routing requests having a bandwidth requirement and a number of constraints on additive QoS measures. Two scenarios have been analyzed, the first under loose QoS constraints and the other by the tightening of QoS constraints. For every test of the first scenario the call blocking rate of SAMCRA$B$ was the minimum or very close to the minimum. The simulations therefore revealed that the proposed path length function of SAMCRA-B (based on the current bandwidth availability) allows a considerable advantage over SAMCRA. The second scenario showed that SAMCRA-B performs better and better than the other algorithms (SAMCRA and TE-DB) when decreasing the QoS constraints. SAMCRA, too, reduces its gap from TE-DB as the QoS con- 
straints become more stringent. If we also consider the analysis of path computation times, we can conclude that SAMCRA-B achieves the best performance at a low computational cost.

The scenarios covered by our simulations were necessarily limited and therefore the results only indicate a potential for SAMCRA with a properly chosen path length function. Further investigation is needed to confirm our claim.

\section{Acknowledgments}

This work has been partly supported by the European Union under the ENext Project FP6-506869 and by the "Ministero dell'Istruzione, dell'Universita e della Ricerca (MIUR)" in the framework of the FIRB Project "Middleware for advanced services over large-scale, wired-wireless distributed systems (WEBMINDS)".

The research of Stefano Avallone is partly funded by the European Doctoral School of Advanced Topics in Networking (SATIN), the instrument employed by the E-Next Network of Excellence to invest in education of researchers for the European Research Area.

\section{References}

Ahuja, R.K., Magnanti, T.L., and Orlin, J.B. (1993). Network Flows: Theory, Algorithms and Applications. Englewood Cliffs, NJ: Prentice-Hall.

Banerjee, G. and Sidhu, D. (2002). Comparative analysis of path computation techniques for MPLS traffic engineering. Computer Networks, 40:149-165.

De Neve, H. and Van Mieghem, P. (2000). TAMCRA: A Tunable Accuracy Multiple Constraints Routing Algorithm. Computer Communications, 23:667-679.

Guerin, R., Williams, D., and Orda, A. (1997). QoS routing mechanisms and OSPF extensions. In Proc. Globecom.

Iliadis, I. and Bauer, D. (2002). A New Class of Online Minimum-Interference Routing Algorithms. Networking 2002, LNCS 2345, pages 959-971.

Kar, K., Kodialam, M., and Lakshman, T.V. (2000). Minimum Interference Routing of Bandwidth Guaranteed Tunnels with MPLS Traffic Engineering Applications. IEEE Journal on Selected Areas in Communications, 18(12):2566-2579.

Kuipers, F., Van Mieghem, P., Korkmaz, T., and Krunz, M. (2002). An overview of constraintbased path selection algorithms for QoS routing. IEEE Communications Magazine, 40(12):50 55.

Medina, A., Matta, I., and Byers, J. (2000). On the Origin of Power Laws in Internet Topology. ACM Computer Communications Review, 30(2).

Van Mieghem, P. and Kuipers, F.A. (2004). Concepts of Exact QoS Routing Algorithms. IEEE ACM Transactions on Networking, 12(5):851-864.

Wang, Bin, Su, Xu, and Chen, C.L.P. (2002). A New Bandwidth Guaranteed Routing Algorithm for MPLS Traffic Engineering. In Proc. of IEEE International Conference on Communications, ICC 2002 . 